\title{
Multislice computed tomography/contrast- enhanced ultrasound image fusion as a tool for evaluating unclear renal cysts
}

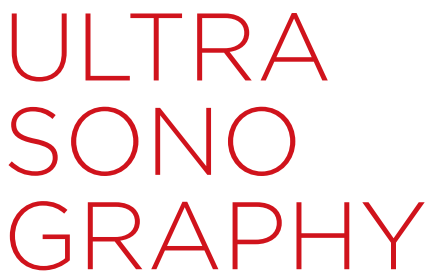

\author{
Johannes Rübenthaler ${ }^{1}$, Stephanie Wilson ${ }^{2}$, Dirk-André Clevert ${ }^{1}$ \\ 'Department of Clinical Radiology, Interdisciplinary Ultrasound Center, Ludwig-Maximilians- \\ University of Munich-Grosshadern Campus, Munich, Germany; ${ }^{2}$ Department of Radiology, \\ University of Calgary, Foothills Medical Centre, Calgary, Canada
}

\section{TECHNICAL NOTE}

https://doi.org/10.14366/usg. 18024 pISSN: 2288-5919 • elSSN: 2288-5943 Ultrasonography 2019;38:181-187

Ultrasonography is a generally accepted imaging technique for diagnosing and monitoring cystic renal lesions. The widely used Bosniak classification (I-IV) categorizes renal cystic lesions into five distinctive groups according to ultrasonography and computed tomography (CT) image criteria. For solid renal lesions, determination of vascularity is discriminatory for malignancy in most instances. In indeterminate cases, contrast-enhanced ultrasound (CEUS) and magnetic resonance imaging/CT-ultrasound image fusion are able to detect and characterize difficult pathologies, with superior performance to either technique alone. In contrast to multislice CT (MS-CT), ultrasound image fusion is a real-time imaging technique that can be used in combination with other cross-sectional imaging modalities. This technical note describes stateof-the-art image fusion of CEUS and MS-CT to detect and characterize unclear renal pathologies.

Keywords: Cystic renal masses; Bosniak classification; Contrast enhanced ultrasound; Image fusion; Multislice-CT

\section{Introduction}

In recent years, the detection and characterization of cystic renal pathologies have increased with the introduction of specialized imaging modalities into normal clinical practice. Furthermore, image fusion and contrast-enhanced ultrasound (CEUS) are implemented in more and more clinical high-end ultrasound systems, and ultrasound contrast agents have officially been approved by the Food and Drug Administration for use in the United States [1]. The most widespread method for the detection and characterization of renal cysts is native B-mode ultrasound, but in some cases renal cysts cannot be sufficiently characterized relying solely on B-mode ultrasound. The addition of image fusion to B-mode ultrasound allows real-time CEUS examinations to be integrated with multislice computed tomography (MS-CT) image datasets, with each component contributing to improved performance. Image fusion is becoming the method of choice for the further characterization of renal pathologies that are unclear on computed tomography (CT), especially in young patients and patients with reduced renal function or other contraindications for contrast-enhanced CT [2]. The main challenge for imaging is to differentiate between simple and complicated cystic renal lesions and cystic and

Received: May 8, 2018

Revised: October 2, 2018

Accepted: October 3, 2018

Correspondence to:

Dirk-André Clevert, MD, Department of Clinical Radiology, Interdisciplinary Ultrasound Center, LudwigMaximilians-University of MunichGrosshadern Campus, Marchioninistr. 15, 81377 Munich, Germany

Tel. +49-89-440073620

Fax. +49-89-440078832

E-mail: Dirk.Clevert@med.unimuenchen.de

This is an Open Access article distributed under the terms of the Creative Commons Attribution NonCommercial License (http://creativecommons.org/ licenses/by-nc/4.0/) which permits unrestricted noncommercial use, distribution, and reproduction in any medium, provided the original work is properly cited.

Copyright (? 2019 Korean Society of Ultrasound in Medicine (KSUM)

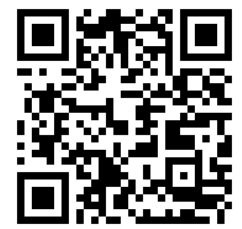

How to cite this article:

Rübenthaler J, Wilson S, Clevert DA Multislice computed tomography/contrastenhanced ultrasound image fusion as a tool for evaluating unclear renal cysts. Ultrasonography. 2019 Apr;38(2):181-187. 
solid renal tumors.

About $6 \%$ of all renal lesions that do not show classical clinical signs or symptoms are cystic renal cell carcinomas [3]. Between 1996 and 2004, renal tumors showed the highest mortality rate of all tumors of the urinary tract in the United States [4]. Renal cell carcinoma is the most common primary malignant neoplasm of the kidney. It is most commonly detected during diagnostic procedures performed for other primary diseases [5]. The therapy of choice is full or partial nephrectomy depending on the location and size of the tumor [6]. In addition to cystic and solid renal tumors, focal cystic lesions are a common pathology of the kidney. The incidence of focal cystic renal lesions shows a positive correlation with age. More cystic renal lesions can be found in elderly patients than in younger patients. By the age of 50 years, roughly $20 \%$ of patients show cystic renal lesions and $50 \%$ of all patients show cystic renal lesions at autopsy. Cystic renal lesions are also differently distributed between the genders, with a male-to-female ratio of 2:1 [7]. This technical note describes state-of-the-art image fusion with CEUS and MS-CT to detect and characterize unclear renal pathologies.

\section{Materials and Methods}

\section{Native B-Mode Sonography, CEUS, and Image Fusion}

All cases presented in this article were assessed either with a Philips EPIQ 7 (Philips Medical Systems, Bothell, WA, USA) high-end ultrasound system using a 2-9-MHz curved array multi-frequency transducer or with a GE E9 (GE Healthcare, Milwaukee, WI, USA) system using a 1-5-MHz curved array multi-frequency transducer. We retrospectively evaluated 1,251 CEUS examinations of kidneys performed between 2005 and 2015, and selected seven patients with additional image fusion datasets to show the spectrum of advantages provided by this technique. All patients provided oral and written informed consent. Institutional Review Board approval was granted for the use of all data in this study. All examinations were initially performed in native B-mode ultrasound. Native B-mode ultrasonography is widely accepted as the standard examination in the diagnostic evaluation of the kidneys [8-12]. After the first round of B-mode ultrasonography, all cases of focal renal pathology were then examined with CEUS. Prior to the ultrasound contrast agent examination, all patients provided oral and written informed consent. CEUS was carried out using contrast mode in the corresponding software; this software mode offers a contrast agent protocol with a low mechanical index that detects and processes the non-linear signals echoed by the microbubbles of the contrast agent and generates a visual separation between tissue and contrast agent signals to obtain better diagnostic information [13]. The ultrasound systems feature high-performance processors and allow the documentation of dynamic image sequences in cine mode. After intravenous injection of the contrast agent, the microbubbles oscillate in response to the ultrasound waves and reflect an echo on the same wavelength but with an increased echo intensity of up to $30 \mathrm{~dB}$, which is a signal intensity roughly 1,000 times higher than is initially transmitted [14].

Modern second-generation ultrasound contrast agents (e.g., Lumason, Bracco Diagnostics Inc., Monroe Township, NJ, USA [for the USA]/SonoVue, Bracco, Milan, Italy [for the rest of the world]) consist of a shell of phospholipids that stabilize the core of microbubbles containing sulfur hexafluoride gas (SF-6) [15]. After administration, the contrast agent is eliminated within a short time period. The phospholipids are metabolized via an endogenous mechanism. The SF- 6 gas is eliminated through the lungs within a time period of 4 to 8 minutes. Thereby, the ultrasound contrast agent does not interfere with renal or thyroid function. With a size of only 2-10 $\mu \mathrm{m}$, each microbubble is about the same size as a single erythrocyte. Unlike CT and magnetic resonance imaging contrast agents, ultrasound contrast agents do not diffuse into the interstitial space [16]. As purely intravascular contrast agents, they can be described as blood-pool contrast agents that only show vascularized tissue [17]. For diagnostic views of the kidney, 1.0-1.5 $\mathrm{mL}$ of ultrasound contrast agent is administered as a bolus injection intravenously, followed by a flush of $10 \mathrm{~mL}$ saline solution $(0.9 \%$ $\mathrm{NaCl}$ ). As with other contrast agents, the major side effects of the contrast agent must be considered as part of daily clinical practice. The most important side effect of ultrasound contrast agents is an anaphylactic reaction, which occurs in 1 in 10,000 cases [18].

\section{Image Fusion}

For complicated renal pathologies, real-time image fusion can be performed with high-end ultrasound systems. This is especially useful in young patients and patients with unclear CT findings, reducing their exposure to radiation as well as to nephrotoxic contrast agents $[19,20]$. The hardware of the ultrasound system needs an additional magnetic field generator and a position sensor for the ultrasound transducer to perform image fusion. The position sensor makes it possible to detect the position of the transducer in the 3-dimensional room. Image fusion is possible with all other imaging modalities, including CT. Digital Imaging and Communications in Medicine (DICOM) datasets of CT scans can be co-registered with the help of the ultrasound system software and can be viewed in a side-by-side mode or in an overlay mode in real time. During image fusion, it is still technically possible to use all other image modes of the ultrasound system, such as color Doppler and CEUS. Using all the different image techniques in real time allows comprehensive imaging and characterization of the vascularization 
of renal lesions $[11,21-23]$. Lesions that cannot be sufficiently seen on native B-mode ultrasound, for example due to a very deep location of the lesion, sometimes cannot be co-registered with the CT imaging dataset due to the normal limitations of conventional ultrasound. To perform image fusion with imaging series from different modalities that were acquired at different time points, it is mandatory to perform spatial co-registration to be sure that the pixels from each dataset are approximately co-registered with the same volume. One method to achieve correct co-registration is to define several standard points of registration, which normally are common anatomic structures. In case of the kidney, this can be most easily done using the kidney artery as a landmark or using special landmarks, such as simple renal cysts. Most ultrasound systems use a rigid transformation matrix for image fusion, because these matrices are easy to handle. A main disadvantage of this technique is that there is no compensation for movement caused by the patient's breathing or other motions, which can cause unsuccessful co-registration of the imaging datasets. To avoid misalignment of the fused imaging datasets, it can be helpful to ask the patient to breathe slowly, if possible. To further improve the alignment of the imaging data sets, it can be helpful to scan in the same respiratory phase as the CT imaging dataset, which normally is in inspiration breath-hold, and to scan in the same position as when the CT imaging datasets were acquired [24]. If possible, the CT imaging datasets should be evaluated before performing image fusion to determine which CT phase should be used as an imaging dataset for the fusion.

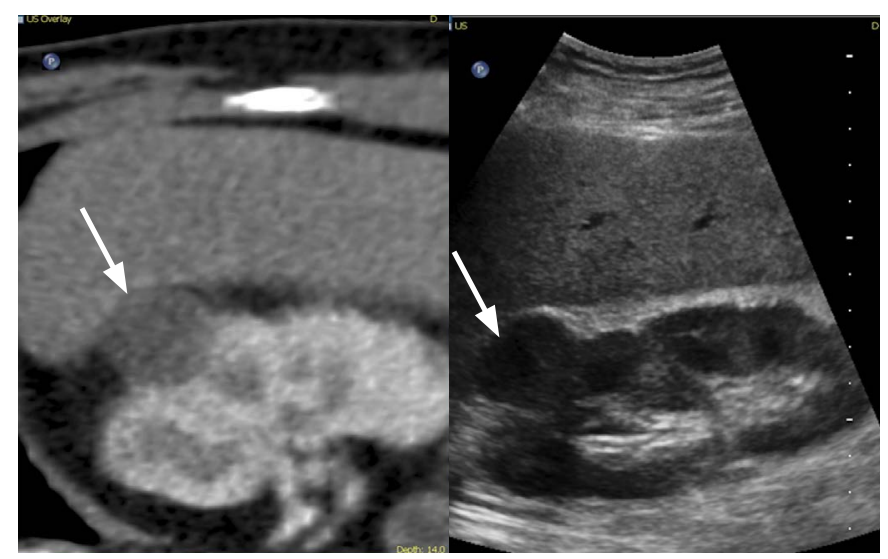

A

Fig. 2. Real-time side-by-side image fusion of a patient with a mildly complex benign cortical renal cyst seen on computed tomography (CT) and ultrasound.

A. The initial CT report stated that the renal cyst density was elevated, and additional ultrasound for further evaluation was recommended. With image fusion, the relevant cyst (arrows) on CT can be easily identified on native B-mode ultrasound, showing a slightly increased intracystic echogenicity, suggesting a Bosniak category II cyst. B. Additional real-time side-by-side contrast-enhanced ultrasound (CEUS) of the renal cystic lesion (arrows) shows the CT imaging data set and real-time CEUS. With the help of CEUS, no vascularization of the cortical cyst could be visualized, confirming the classification as a Bosniak category II cyst.

e-ultrasonography.org

Ultrasonography 38(2), April 2019 


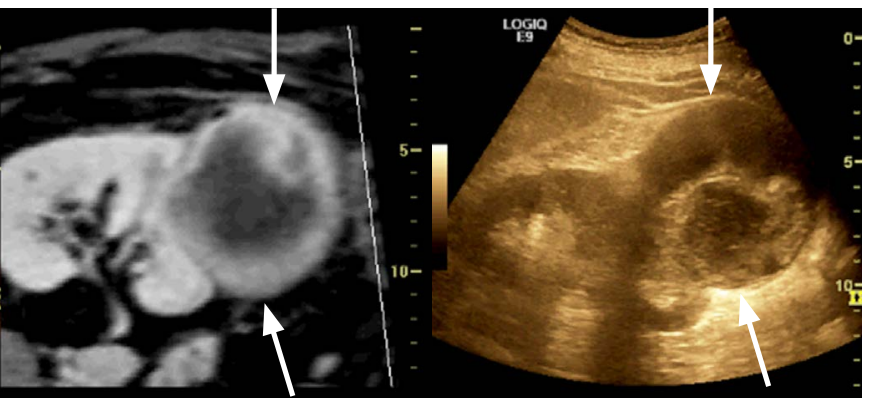

A

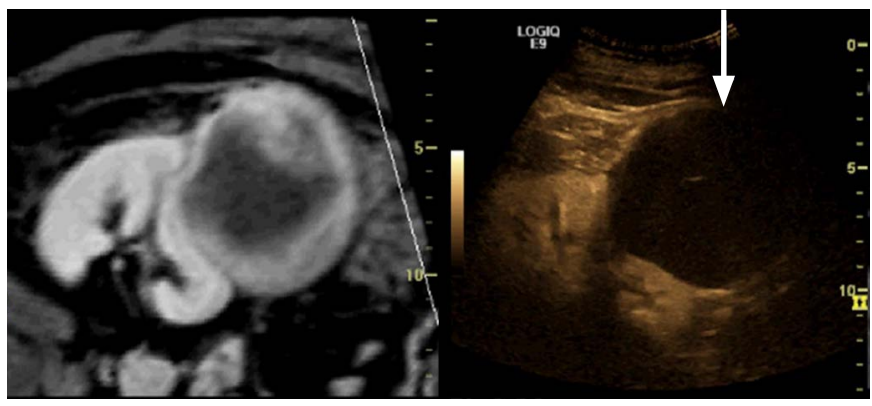

B

Fig. 3. Fusion of a moderately complex subcapsular cyst with computed tomography (CT) and ultrasound.

A. On the real-time fused images, multiple septa and thickened walls (arrows) can be seen on both CT and native B-mode ultrasound, in line with the features of a Bosniak category IV cyst, suggesting a malignant tumor. B. On the real-time image fusion with fused CT and contrastenhanced ultrasound data set, there is no major vascularization of the septa or the thickened walls (arrow), drastically changing the Bosniak category from IV to IIF and significantly impacting the patient's management. The intracystic septa were likely caused by an intracystic hemorrhage. Despite the classification as a Bosniak IIF cyst, the patient wanted the lesion to be surgically removed, and the histopathological findings did not show any signs of malignancy.

excellent inter-rater reliability of the Bosniak criteria [25]. The Bosniak classification is also used with other imaging modalities, such as CEUS. Image fusion with CEUS can sometimes be a superior imaging method for the discovery and classification of cystic renal lesions [26].

Renal cystic lesions are characterized according to their morphological appearance into five different categories (Bosniak categories I-IV).

Bosniak category I cysts are round to oval on native B-mode ultrasound and show a thin wall without any septa or calcifications. The density of the cysts is mostly equal to water [6]. Renal cystic lesions deviating from these criteria are characterized according to their morphological appearance into four other groups (Bosniak categories II, IIF, III, and IV) [6,27]. While Bosniak category I, II, and IV cysts are mostly easily to characterize using solely $\mathrm{CT}$ or ultrasound imaging criteria, Bosniak category IIF and III cysts remain a challenge for imaging; however, this challenge can be overcome by using information from different imaging modalities in real time with image fusion.

\section{Category I}

Category I cysts are benign or simple cysts with thin walls and without any septa or calcifications. Cysts of this category do not reveal solid components or contrast enhancement on ultrasound and are easy to characterize solely using CT or ultrasound criteria [28] (Fig. 1).

\section{Category II}

Category II cysts can have septa thinner than $1 \mathrm{~mm}$, and optionally these septa can be lightly calcified. These cysts are still

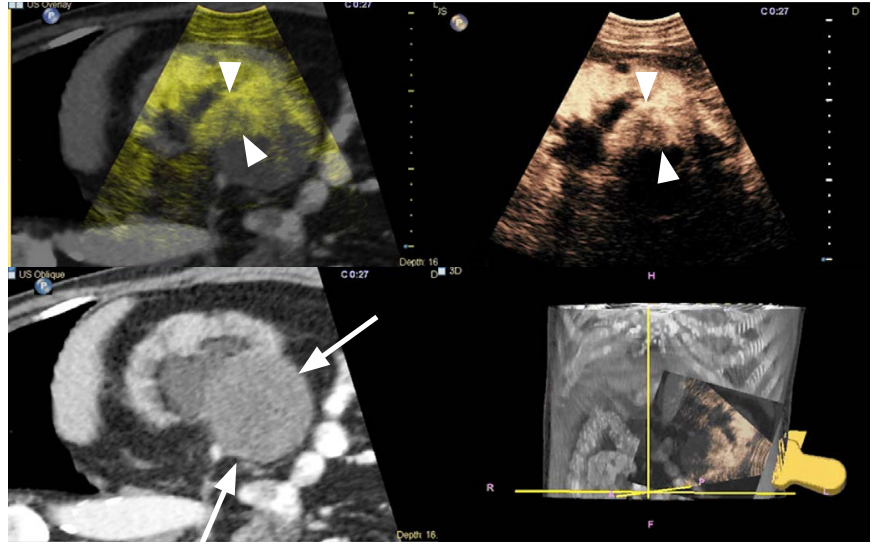

Fig. 4. Real-time contrast-enhanced ultrasound image fusion in a patient with an unclear renal cystic lesion. The imaging software of the image fusion mode shows four different pictures: a computed tomography (CT)/contrast-enhanced ultrasonography (CEUS) real-time overlay mode (upper left), the CEUS real-time image (upper right), the $\mathrm{CT}$ imaging dataset (lower left), and a real-time 3-dimensional navigation image fusion of the ultrasound image and the $\mathrm{CT}$ dataset (lower right). On the initial CT report, this cystic lesion was reported to have elevated density (arrows) and slightly thickened and contrast-enhancing walls, and Bosniak category IIF was suggested as the diagnosis. Additionally, hydronephrosis was reported and additional ultrasound for further evaluation was recommended. CEUS shows a vascularized part of this cystic lesion with increased contrast uptake (arrowheads), which can also be seen in CT/CEUS real-time overlay mode and clearly extends over the minimally thickened wall visualized in the CT examination (arrowheads). The CEUS image fusion examination upgraded this lesion from Bosniak category IIF to Bosniak category IV, and the histological work-up after surgical resection revealed a papillary renal cell carcinoma. 


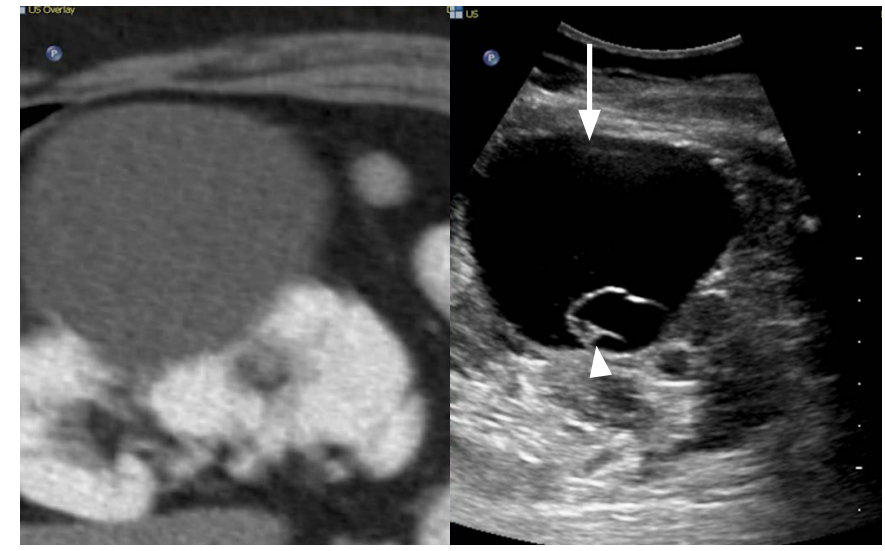

A

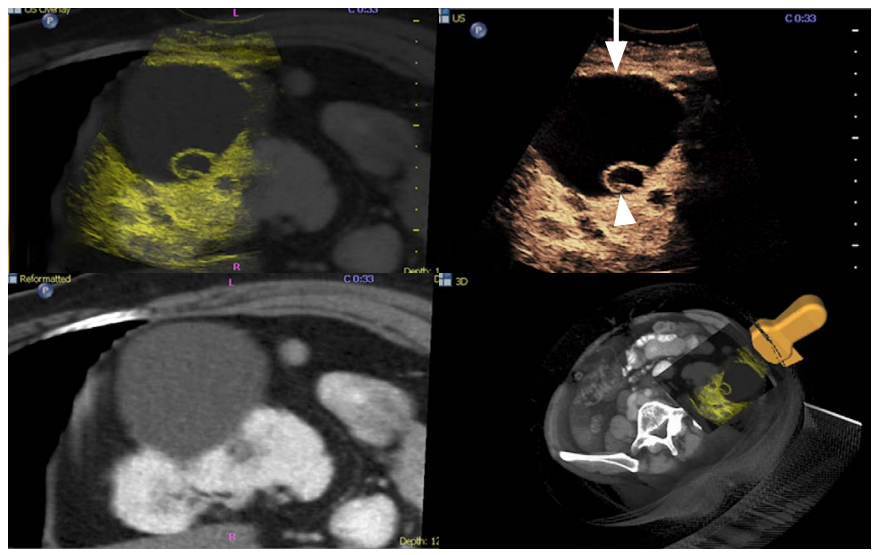

B

Fig. 5. Image fusion of an indeterminate complex renal cyst seen on computed tomography (CT) with ultrasound.

A. The initial CT report suggested a Bosniak category II cyst and because of the size of the cyst, and the fused B-mode ultrasound shows thickened wall (arrow) and multiple septa (arrowhead). B. Real-time contrast-enhanced ultrasound image fusion shows four different pictures: a computed tomography (CT)/contrast-enhanced ultrasonography (CEUS) real-time overlay mode (upper left), the CEUS real-time image (upper right), the $\mathrm{CT}$ imaging dataset (lower left), and a real-time 3-dimensional navigation image fusion of the ultrasound image and the CT dataset (lower right). The CT/CEUS real-time overlay mode and CEUS show major vascularization of the thickened wall (arrow) and septa (arrowhead), which cannot be seen solely relying on the CT imaging data set. The CEUS image fusion examination upgraded this lesion from the initial Bosniak category II to Bosniak category III because of the vascularization without solid components, and the histological work-up after surgical resection revealed a clear-cell renal cell carcinoma.

easily identifiable using solely CT or ultrasound imaging criteria. Homogenous lesions measuring less than $3 \mathrm{~cm}$ and showing sharp margins without contrast enhancement also belong to this category [27,28] (Fig. 2).

\section{Category IIF}

Category IIF cysts are those that cannot be characterized as category II or as category III. These are complicated cysts that need to receive periodic follow-up.

The characteristics of these cysts are multiple septa that can show minimally thickened walls and discrete contrast enhancement of the walls or septa [28]. Category IIF cysts can also show thin or thick calcifications. Normally, these changes are of benign origin, but periodic follow-up is necessary to detect possible malignant degeneration [29]. Bosniak IIF cysts seen on MS-CT are desirable candidates for evaluation using CEUS image fusion, because this examination might downgrade or upgrade the initial classification based solely upon the CT imaging findings (Figs. 3, 4).

\section{Category III}

Category III cysts are lesions of unclear malignant potential that cannot be categorized as belonging the other categories with the help of $\mathrm{CT}$ or ultrasound. The potential malignancy of these lesions must be invasively evaluated. Characteristics of these cysts include a homogenous or irregular thickening of the walls and/or septa

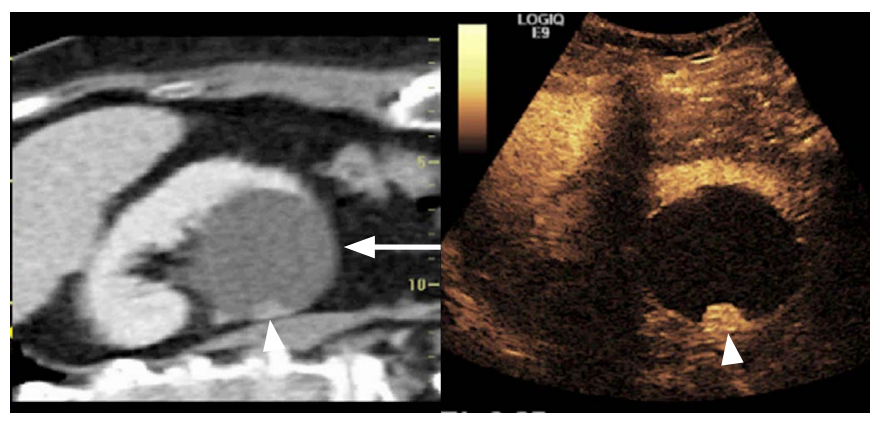

Fig. 6. Real-time side-by-side image fusion of a complex renal cystic mass using computed tomography (CT) and contrastenhanced ultrasound (CEUS). The initial CT (left) shows a cyst (arrow) with focal irregular thickened contrast-enhancing wall nodule (arrowhead) that is also clearly visualized using CEUS (right). This cystic lesion was initially reported as Bosniak category III, but the additionally performed CEUS image fusion examination clearly reveals a solid hypervascularized nodule (arrowhead) in line with a Bosniak category IV cyst.

and multiple contrast-enhancing septa [27-29]. The malignancy rate of category III cysts is about 50\%. Image fusion can be helpful when evaluating these cysts, as the dynamic perfusion information gathered through CEUS might change the classification (Fig. 5).

\section{Category IV}

Category IV cysts show a malignancy rate of nearly 100\%. The 


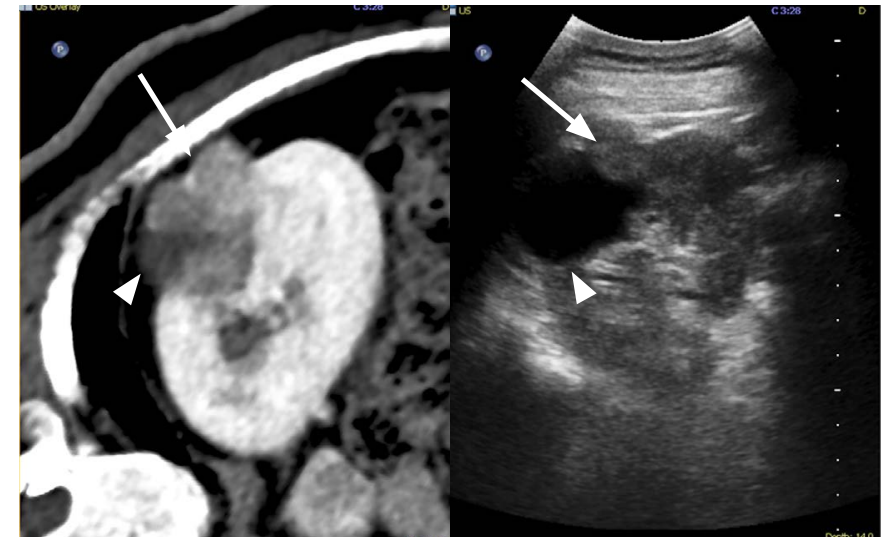

A

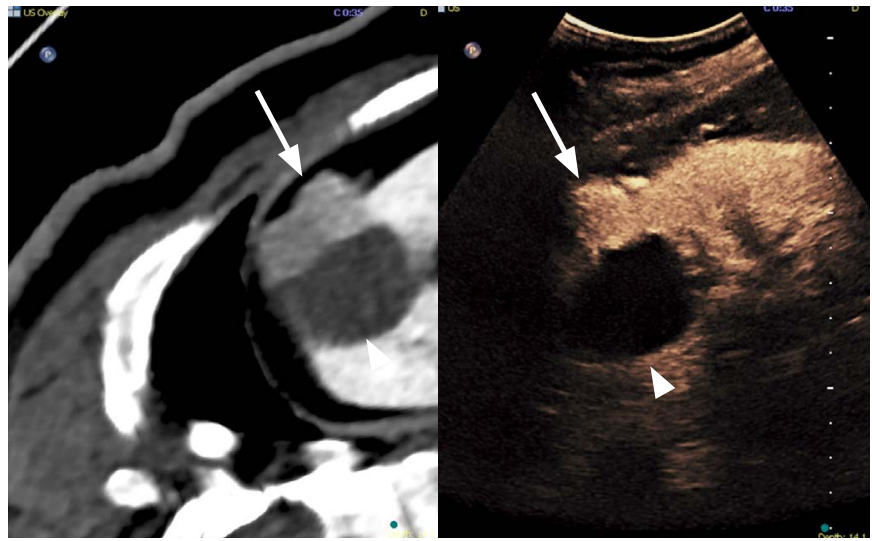

B

Fig. 7. Image fusion of a partly cystic renal mass lesion showing solid and cystic components in computed tomography (CT) and ultrasound.

A. Real-time side-by-side image fusion of CT (left) and B-mode ultrasound (right) shows solid (arrows) and cystic (arrowheads) components suggesting a Bosniak category IV cyst. B. On an additional real-time side-by-side image fusion using CT/contrast-enhanced ultrasound (CEUS) side-by-side mode, the solid components (arrows) show major vascularization on CT and CEUS, whereas the cystic components (arrowheads) do not show major vascularization. The lesion shows the typical features of a Bosniak category IV cyst on CT and on CEUS, and the histological workup after surgical removal revealed a clear-cell renal cell carcinoma.

characteristics of category IV cysts are similar to those of category III, but they also show irregular contrast-enhancing soft tissue components and additional solid components on ultrasound [2729]. Their diagnosis can mostly be made using CT or ultrasound imaging criteria. Characterization using invasive imaging techniques or biopsy is usually necessary (Figs. 6, 7).

\section{Discussion}

Native B-mode ultrasonography and CEUS with additional image fusion are uncomplicated, precise, and fast imaging methods for the characterization of typical and atypical renal lesions. All imaging methods play a fundamental role in the identification and characterization of renal cysts. Because CEUS can acquire realtime imaging data, it is possible to use CEUS to obtain additional knowledge about the vascularization of renal lesions that might downgrade or upgrade the initial categorization, which can have a serious impact on patient management and follow-up [30]. Additionally, image fusion options are currently making it possible to easily detect, image, and characterize unclear renal pathologies and to interpret images between different imaging modalities. CEUS and image fusion are especially beneficial for younger patients to reduce their exposure to radiation in follow-up examinations and in older patients to minimize their exposure to nephrotoxic contrast agents. Finally, the combination of different cross-sectional imaging techniques can enable unclear renal lesions to be resolved, thereby improving decisions regarding treatment. Image fusion can also be used for needle guidance in ultrasound interventions, such as for the radiofrequency ablation of renal lesions. Image fusion allows the real-time visualization of the needle-tip position of an interventional needle and makes it possible to track needle positioning in real time and to directly control interventional success using CEUS.

In conclusion, image fusion of renal lesions is a fast and practical technique for the further evaluation and characterization of lesions of uncertain malignant potential, and it can be easily performed in almost all cases during routine clinical practice. Image fusion should always be considered as an additional tool in these cases.

ORCID: Johannes Rübenthaler: https://orcid.org/0000-0003-0832-5662; Stephanie Wilson: https://orcid.org/0000-0001-6396-7612; Dirk-André Clevert: https://orcid. org/0000-0003-3889-5447

\section{Conflict of Interest}

No potential conflict of interest relevant to this article was reported.

\section{References}

1. Seitz K, Strobel D. A milestone: approval of CEUS for diagnostic liver imaging in adults and children in the USA. Ultraschall Med 2016;37:229-232.

2. Nolsoe CP, Lorentzen T. International guidelines for contrastenhanced ultrasonography: ultrasound imaging in the new millennium. Ultrasonography 2016;35:89-103. 
3. Warren KS, McFarlane J. The Bosniak classification of renal cystic masses. BJU Int 2005;95:939-942.

4. Ascenti G, Mazziotti S, Zimbaro G, Settineri N, Magno C, Melloni D, et al. Complex cystic renal masses: characterization with contrastenhanced US. Radiology 2007;243:158-165.

5. Bauer A, Solbiati L, Weissman N. Ultrasound imaging with SonoVue: low mechanical index real-time imaging. Acad Radiol 2002;9 Suppl 2:S282-S284.

6. Bosniak MA. The current radiological approach to renal cysts. Radiology 1986;158:1-10.

7. Ravine D, Gibson RN, Donlan J, Sheffield LJ. An ultrasound renal cyst prevalence survey: specificity data for inherited renal cystic diseases. Am J Kidney Dis 1993;22:803-807.

8. Stock K, Kubler H, Maurer T, Weiss D, Weskott HP, Heemann U. Innovative ultrasound: contrast-enhanced ultrasound of the kidneys. Aktuelle Urol 2017;48:120-126.

9. Putz FJ, Erlmeier A, Wiesinger I, Verloh N, Stroszczynski C, Banas $B$, et al. Contrast-enhanced ultrasound (CEUS) in renal imaging at an interdisciplinary ultrasound centre: Possibilities of dynamic microvascularisation and perfusion. Clin Hemorheol Microcirc 2017;66:293-302.

10. Muller-Peltzer K, Negrao de Figueiredo G, Schwarze V, Rubenthaler J, Clevert DA. Reliable diagnosis of cystic renal lesions. Radiologe 2018;58:887-893.

11. Rubenthaler J, Negrao de Figueiredo G, Mueller-Peltzer K, Clevert $D A$. Evaluation of renal lesions using contrast-enhanced ultrasound (CEUS): a 10-year retrospective European single-centre analysis. Eur Radiol 2018;28:4542-4549.

12. Rubenthaler J, Mueller-Peltzer K, Negrao de Figueiredo G, Gresser E, Clevert DA. CEUS-diagnostic workup of cystic renal lesions. Radiologe 2018;58:545-552.

13. Rubenthaler J, Paprottka KJ, Hameister E, Hoffmann K, Joiko N, Reiser $\mathrm{M}$, et al. Contrast-enhanced ultrasound (CEUS) prediction of focal liver lesions in patients after liver transplantation in comparison to histopathology results. Clin Hemorheol Microcirc 2017;66:303-310.

14. Rubenthaler J, Paprottka KJ, Hameister E, Hoffmann K, Joiko $\mathrm{N}$, Reiser M, et al. Diagnostic accuracy of contrast-enhanced ultrasound (CEUS) in monitoring vascular complications in patients after liver transplantation - diagnostic performance compared with histopathological results. Clin Hemorheol Microcirc 2017;66:311-316.

15. Barr RG. How to develop a contrast-enhanced ultrasound program. J Ultrasound Med 2017;36:1225-1240.

16. Greis C. Technical aspects of contrast-enhanced ultrasound (CEUS) examinations: tips and tricks. Clin Hemorheol Microcirc
2014;58:89-95.

17. Muller-Peltzer K, Rubenthaler J, Reiser M, Clevert DA. Contrastenhanced ultrasound (CEUS) of the liver: critical evaluation of use in clinical routine diagnostics. Radiologe 2017;57:348-355.

18. Wilson SR, Burns PN. Microbubble-enhanced US in body imaging: what role? Radiology 2010;257:24-39.

19. Rubenthaler J, Bogner F, Reiser M, Clevert DA. Contrastenhanced ultrasound (CEUS) of the kidneys by using the Bosniak classification. Ultraschall Med 2016;37:234-251.

20. Rubenthaler J, Paprottka K, D'Anastasi M, Reiser M, Clevert DA. Diagnosis of perinephric retroperitoneal lymphangioma supported by contrast-enhanced ultrasound (CEUS). Clin Hemorheol Microcirc 2017;65:43-47.

21. Rubenthaler J, Paprottka KJ, Marcon J, Reiser M, Clevert DA. MRI and contrast enhanced ultrasound (CEUS) image fusion of renal lesions. Clin Hemorheol Microcirc 2016;64:457-466.

22. Rubenthaler J, Paprottka K, Marcon J, Hameister E, Hoffmann K, Joiko $\mathrm{N}$, et al. Comparison of magnetic resonance imaging (MRI) and contrast-enhanced ultrasound (CEUS) in the evaluation of unclear solid renal lesions. Clin Hemorheol Microcirc 2016;64:757-763.

23. Reimann R, Rubenthaler J, Hristova P, Staehler M, Reiser M, Clevert DA. Characterization of histological subtypes of clear cell renal cell carcinoma using contrast-enhanced ultrasound (CEUS). Clin Hemorheol Microcirc 2015;63:77-87.

24. Ewertsen C, Saftoiu A, Gruionu LG, Karstrup S, Nielsen MB. Realtime image fusion involving diagnostic ultrasound. AJR Am J Roentgenol 2013;200:W249-W255.

25. Curry NS, Cochran ST, Bissada NK. Cystic renal masses: accurate Bosniak classification requires adequate renal CT. AJR Am J Roentgenol 2000;175:339-342.

26. Barr RG, Peterson $C$, Hindi A. Evaluation of indeterminate renal masses with contrast-enhanced US: a diagnostic performance study. Radiology 2014;271:133-142.

27. Radermacher J. Ultrasound of the kidney and renal vessels. I: Normal findings, congenital diseases, diseases of the kidney parenchyma. Internist (Berl) 2003;44:1283-1297.

28. Israel GM, Hindman N, Bosniak MA. Evaluation of cystic renal masses: comparison of $\mathrm{CT}$ and MR imaging by using the Bosniak classification system. Radiology 2004;231:365-371.

29. Clevert DA, Horng A, Staehler M, Haseke N, Stief C, Reiser M. Diagnostic algorithm in cystic renal masses. Urologe $A$ 2010;49:421-431.

30. Cantisani V, Wilson SR. CEUS: where are we in 2015? Eur J Radiol 2015;84:1621-1622. 